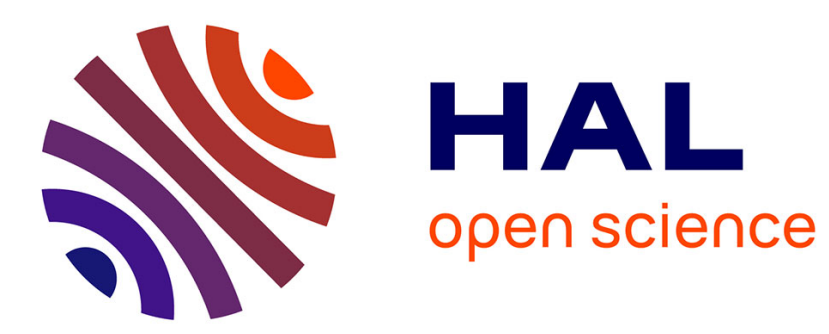

\title{
Une méthode améliorée de mesure de la polarisabilité des atomes libres à l'aide du microscope électronique de champ
}

\author{
J.B. Brosse, J.M. Bermond, M. Drechsler
}

\section{- To cite this version:}

J.B. Brosse, J.M. Bermond, M. Drechsler. Une méthode améliorée de mesure de la polarisabilité des atomes libres à l'aide du microscope électronique de champ. Revue de Physique Appliquée, 1976, 11 (5), pp.677-683. 10.1051/rphysap:01976001105067700 . jpa-00244103

\section{HAL Id: jpa-00244103 https://hal.science/jpa-00244103}

Submitted on 1 Jan 1976

HAL is a multi-disciplinary open access archive for the deposit and dissemination of scientific research documents, whether they are published or not. The documents may come from teaching and research institutions in France or abroad, or from public or private research centers.
L'archive ouverte pluridisciplinaire HAL, est destinée au dépôt et à la diffusion de documents scientifiques de niveau recherche, publiés ou non, émanant des établissements d'enseignement et de recherche français ou étrangers, des laboratoires publics ou privés. 


\title{
UNE MÉTHODE AMÉLIORÉE DE MESURE DE LA POLARISABILITÉ DES ATOMES LIBRES A L'AIDE DU MICROSCOPE ÉLECTRONIQUE DE CHAMP
}

\author{
J. B. BROSSE (*), J. M. BERMOND et M. DRECHSLER
}

Centre de Recherches sur les Mécanismes de la Croissance Cristalline, C. N. R. S. Université d'Aix Marseille III, Faculté des Sciences et Techniques de Saint-Jérôme 13397 Marseille Cedex 4, France

(Reçu le 15 mars 1976, accepté le 21 mai 1976)

\begin{abstract}
Résumé. - Les trajectoires des atomes neutres sont courbées près de la pointe d'un microscope électronique de champ, à cause de leur polarisation par le champ électrique inhomogène. Ce phénomène a permis de déterminer la polarisabilité $\alpha$ des atomes du jet (Drechsler et Müller). Nous avons généralisé la théorie et modifié la méthode de mesure pour améliorer la précision et simplifier l'utilisation. On trouve que la forme des trajectoires dépend uniquement d'un paramètre qui inclut la polarisabilité $(\alpha)$ la masse, la vitesse initiale et le champ électrique au sommet de la pointe. On peut déterminer la polarisabilité si l'on mesure les deux temps nécessaires pour déposer sur une face de la pointe, à l'aide d'un jet de flux constant, le même degré de recouvrement avec et sans champ. La méthode a été appliquée à l'atome de plomb ; on a obtenu : $\alpha=19,5 \pm 6 \AA^{3}$, ce qui est en accord avec les estimations théoriques.

Abstract. - The trajectories of neutral atoms are curved near the tip of a field electron microscope, due to their polarization in the inhomogeneous electric field. This phenomenon has permitted a rough measurement of the polarizability of atoms (Drechsler an Müller). We have now generalized the theory and modified the method of measurement in order to improve the precision and to simplify the application. It is found that the trajectories depend on one parameter which includes the polarizability $(\alpha)$, the mass, the initial velocity of the particle and the electric field strength at the tip apex. $\alpha$ can be determined if one measures the two times which are necessary to obtain the same degree of coverage on one face of the tip by adsorption of the beam atoms with and without field. The degree of coverage is monitored by measuring the field electron current emitted from this face. As an example, the method is used to measure the polarizability of $\mathrm{Pb}$; the result is : $\alpha=19,5 \pm 6 \AA^{3}$ which is in agreement with theoretical estimations.
\end{abstract}

1. Introduction. - La polarisabilité d'un atome est une grandeur fondamentale assez mal connue. Pour les atomes libres, elle est déterminée à partir de la déflexion d'un jet atomique par un champ électrique inhomogène. Pour produire un tel champ, on a d'abord utilisé un fil métallique très fin constituant une des armatures d'un condensateur cylindrique d'axe parallèle à la direction $\mathrm{du}$ jet $[1,2]$. Dans un tel appareillage, la répartition du champ électrique et par conséquent les trajectoires atomiques sont facilement calculables. En revanche, la variation du champ normalement à l'axe est relativement faible $\left(10^{6} \mathrm{~V} / \mathrm{cm}^{2}\right.$ [1] $)$ et la déviation du faisceau est faible aussi et difficile à mesurer. En outre, malgré la bonne précision de ces méthodes ( $5 \%$ environ) les techniques de détection des jets atomiques ont limité

(*) Adresse actuelle : Ecole Nationale Supérieure des Mines de Saint-Etienne, Département de Métallurgie, 42023 SaintEtienne, France. leur emploi à des molécules ou des atomes facilement ionisables $\left(\mathrm{H}_{2}, \mathrm{~K}, \mathrm{Li}, \mathrm{Cs}[1,2]\right)$. C'est pourquoi d'autres auteurs ont utilisé, pour la mesure de la polarisabilité, le champ électrique au voisinage de la pointe d'un microscope électronique de champ [3, 4]. Ce champ élevé, très inhomogène $\left(10^{9} \mathrm{~V} / \mathrm{cm}^{2}\right.$ env.) provoque une déviation importante des trajectoires. En outre, la détection des particules se fait très simplement en repérant (sur l'image d'émission de champ par exemple) les régions de la pointe où elles viennent se condenser. On peut donc utiliser tous les corps dont l'adsorption sur la surface de la pointe entraîne un changement du travail de sortie. Ainsi, on a pu mesurer la polarisabilité non seulement de métaux alcalins ( $\mathrm{Ba}, \mathrm{Li}, \mathrm{Cs})$ [3] mais aussi de molécules organiques [3] et de métaux comme $\mathrm{Cu}, \mathrm{W}, \mathrm{Mo}, \mathrm{Ta}$ [4]. En revanche, la distribution du champ électrique au voisinage de la pointe rend très difficile le calcul des trajectoires. Par conséquent, pour déterminer la polarisabilité $(\alpha)$ à partir des don- 
nées expérimentales, on doit utiliser soit une formule approchée semi-empirique [4], soit une méthode graphique par essai et erreur [3], longue et peu précise, qui doit être recommencée pour chaque type de particule. De plus, on suppose toujours que le champ électrique au voisinage de la pointe est celui d'un hyperboloïde de révolution, approximation trop grossière en pratique. Au total, l'erreur sur $\alpha$ doit être au moins de $40 \%$.

Pour profiter du champ d'application très grand de la méthode par microscopie électronique de champ, il nous a semblé possible de formuler la théorie de cette méthode de façon à rendre son utilisation générale (forme de la pointe, type de particule) et relativement aisée. Ceci nous a conduit aussi à simplifier et généraliser la méthode expérimentale pour mesurer $\alpha$ et, par conséquent, à diminuer l'erreur sur cette grandeur. Ces améliorations sont décrites dans cet article. Nous commençons par établir une propriété générale des trajectoires de particules polarisables dans un champ électrique. Nous appliquons ensuite cette propriété au tracé d'abaques résumant les caractéristiques de telles trajectoires dans le champ de la pointe d'un M. E. C. quelle que soit la nature des particules. Au moyen de ces abaques, la polarisabilité d'une particule quelconque peut être déterminée par une mesure simple dont nous donnons la méthode.

2. Une propriété générale des trajectoires de particules polarisables dans un champ électrique. - Une particule de masse $m$ et de polarisabilité $\alpha$, placée dans un champ électrique $\mathbf{E}$ est soumise à une force :

$$
\mathbf{f}=\frac{1}{2} \alpha \operatorname{grad} \mathbf{E}^{2} .
$$

Si l'énergie potentielle de la particule est prise nulle en un point où le champ est nul, cette particule possède alors dans le champ une énergie potentielle due à la polarisabilité :

$$
W=-\frac{1}{2} \alpha E^{2} .
$$

La trajectoire de la particule est déterminée par :

- l'équation du mouvement

$$
m \frac{\mathrm{d} \mathbf{v}}{\mathrm{d} t}=\frac{1}{2} \alpha \operatorname{grad} \mathbf{E}^{2}
$$

- le point de départ $M_{0}$ et la vitesse initiale $\mathbf{v}_{0}$.

L'énergie totale $W$ de la particule est conservative. Si le champ est nul en $M_{0}$, on a en tout point de l'espace :

$$
W=\frac{1}{2} m v_{0}^{2}=\frac{1}{2} m v^{2}-\frac{1}{2} \alpha E^{2} .
$$

D'autre part, si $E_{0}$ est le module du champ en un point fixe arbitraire (où $\mathbf{E} \neq 0$ ), le champ en tout point $M$ s'exprime par : $\mathbf{E}=E_{0} \gamma(M)$, où $\gamma(M)$ est un vecteur fonction du point $M$ qui ne dépend que de la géométrie des électrodes.
En posant $\lambda=\alpha E_{0}^{2} / m v_{0}^{2}$, l'équation (1) et son intégrale première (2) s'écrivent alors respectivement:

$$
\begin{gathered}
\frac{\mathrm{d} \mathbf{v}}{\mathrm{d} t}=\frac{v_{0}^{2}}{2} \lambda \operatorname{grad} \gamma^{2} \\
v^{2}=v_{0}^{2}\left(1+\lambda \gamma^{2}\right)=v_{0}^{2}|\Phi(M, \lambda)|^{2}
\end{gathered}
$$

avec

$$
\Phi(M, \lambda)=\left|1+\lambda \gamma^{2}\right|^{1 / 2} .
$$

Si l'on considère maintenant les coordonnées $x, y, z$, d'un point $M$ quelconque de la trajectoire comme fonctions de l'abscisse curviligne $s$ de ce point, on peut établir les équations différentielles liant $x, y, z$, et $s$. Par exemple, en projetant (3) sur $O x$ et en utilisant (4) on obtient :

$$
\Phi^{2} \frac{\mathrm{d}^{2} x}{\mathrm{~d} s^{2}}+\Phi \frac{\mathrm{d} \Phi}{\mathrm{d} s} \frac{\mathrm{d} x}{\mathrm{~d} s}=\frac{1}{2} \lambda \frac{\partial \gamma^{2}}{\partial x}
$$

$\Phi, \mathrm{d} \Phi / \mathrm{d} s$, et le second membre de cette équation sont des fonctions composées de $s$ par l'intermédiaire de $x, y$, $z$. $x$ et $s$ sont donc liées par une équation différentielle du second ordre. Il en va de même pour $y$ et $s$, et pour $z$ et $s$.

Il résulte alors des propriétés des équations différentielles du second ordre que, pour $\gamma(M)$ donné, la trajectoire dépend seulement du paramètre $\lambda$, du point de départ $M_{0}$ et des quantités $(\mathrm{d} x / \mathrm{d} s)_{0},(\mathrm{~d} y / \mathrm{d} s)_{0}$, $(\mathrm{d} z / \mathrm{d} s)_{0}$, qui définissent la direction du vecteur vitesse initiale $\mathbf{v}_{0}$. En d'autres termes, pour une géométrie donnée des électrodes et pour une direction donnée de la vitesse initiale, la trajectoire d'une particule ne dépend que de son point de départ et de la quantité $\lambda=\alpha E_{0}^{2} / m V_{0}^{2}$. Bien que cette quantité apparaisse déjà dans des calculs faits par d'autres auteurs sur des géométries sphériques [5], notre calcul montre que la propriété énoncée ci-dessus est générale.

Elle permet de simplifier beaucoup l'étude des trajectoires en fonction de la nature des particules et de la valeur du champ électrique. Dans le paragraphe suivant, nous en donnons un exemple en présentant deux abaques qui résument les caractéristiques des trajectoires au voisinage de la pointe d'un microscope électronique de champ pour des particules polarisables quelconques.

3. Application aux trajectoires de particules polarisables dans un microscope électronique de champ. 3.1 PRINCIPE DE L'eXPÉRIENCE. - Le principe de l'expérience est rappelé par la figure 1 . Un jet moléculaire est issu d'une source située à droite et à l'extérieur de la figure. Les trajectoires, rectilignes en l'absence de champ, sont courbées par le champ qui règne autour de l'apex de la pointe. Leur forme dépend de la polarisabilité des particules. On peut déterminer $\alpha$ à partir de leur tracé [3].

Le potentiel au voisinage de la pointe peut être décrit par le modèle connu de superposition d'une sphère et 


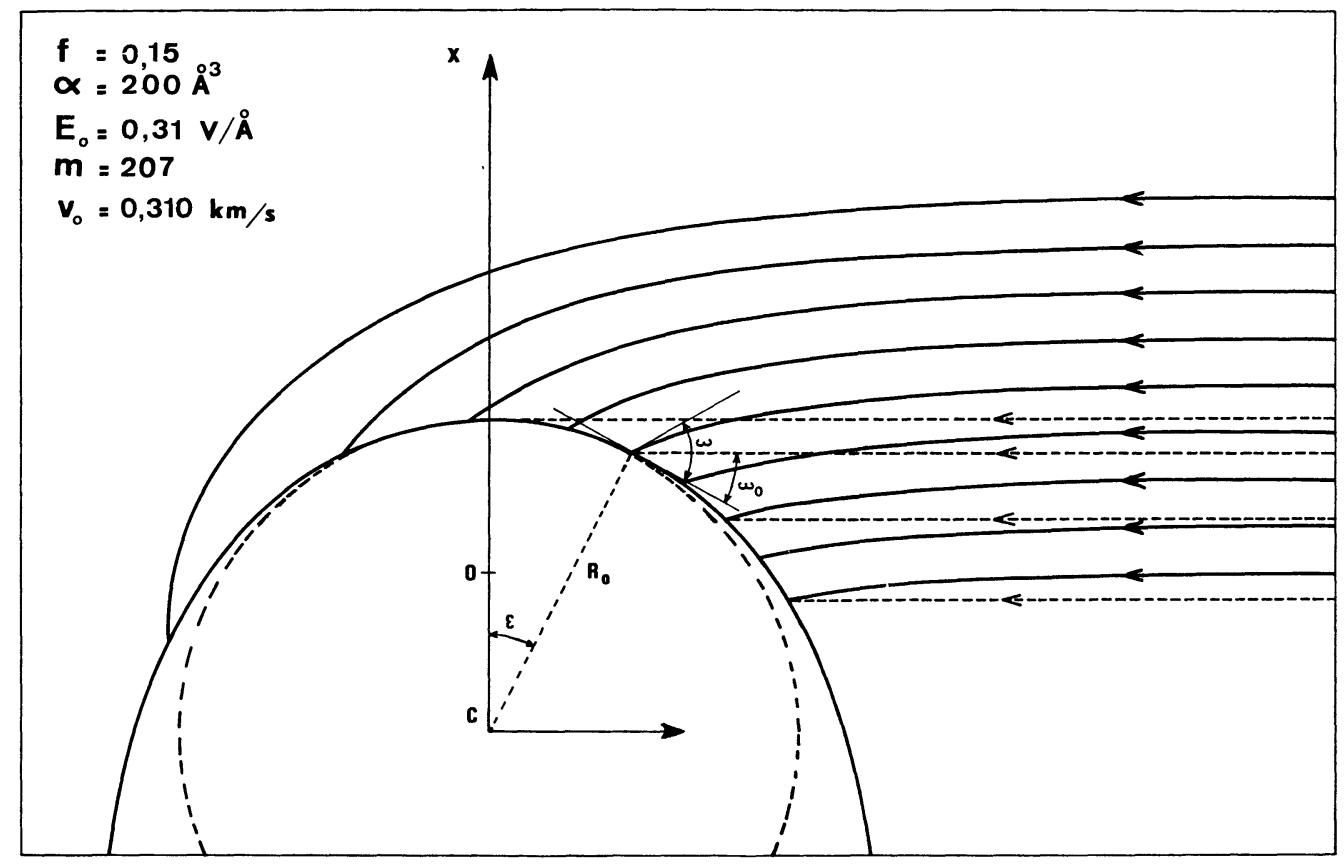

FIG. 1. - Trajectoires des particules du jet avec champ (traits pleins) et sans champ (traits pointillés) près de l'apex d'une pointe émettrice. Dans cet exemple, $\lambda$ selon l'équation (5) est égal à 200.

d'un hyperboloïde [6]. Ce modèle fait apparaître des surfaces équipotentielles caractérisées par un facteur de forme $f$, appelé $\alpha$ dans les autres publications [6], qui représentent bien les formes des pointes utilisées en pratique.

Dans ce type d'expériences, la géométrie des électrodes et la direction de $\mathbf{v}_{0}$ (direction du jet en l'absenc: de champ) sont fixées. On peut prendre pour $E_{0}$ la valeur du champ électrique à l'apex de la pointe, gran . deur qui est expérimentalement accessible [6]. Il suffit de déterminer la forme de la trajectoire en fonction de $M_{0}$ et de $\lambda$ pour être capable de traiter l'ensemble des cas pratiques. En effet, pour une particule quelconque, on peut toujours ajuster les paramètres $E_{0}$ et $v_{0}$ pour se ramener à une valeur de $\lambda$ donnée.

3.2 CALCUL DES TRAJECTOIRES SUR ORDINATEUR. Nous nous sommes limités à l'étude des trajectoires dans le plan de symétrie constitué par la pointe et le jet de particules, c'est-à-dire le plan défini par l'axe de la pointe et le point de départ. Cette restriction du problème est discutée au paragraphe 4.3. Expérimentalement, on peut toujours se ramener à cette situation par le choix des faces cristallines de la pointe sur lesquelles on effectue les mesures.

L'équation différentielle du mouvement (3) n'est pas intégrable par des méthodes simples; il faut donc utiliser une méthode de résolution approchée. A l'aide d'un ordinateur, nous avons tracé les trajectoires, point par point, par la méthode du rayon de courbure.

La position de la particule est repérée à l'aide de coordonnées cartésiennes : l'origine est fixée au centre de courbure du sommet de la pointe (Fig. 1), l'axe des abscisses coïncide avec l'axe de la pointe, et l'unité sur cet axe est le rayon $R_{0}$ au sommet de la pointe.

L'ordonnée du point de départ est fixée à une distance $(25000 \AA)$ où l'intensité du champ électrique est relativement faible, et la trajectoire des particules est encore pratiquement rectiligne. Les trajectoires initiales peuvent donc être considérées comme parallèles.

En chaque point de la trajectoire, l'ordinateur calcule :

- le potentiel,

- la vitesse de la particule,

- les composantes de la force,

- le rayon de courbure,

- la direction de la tangente,

- les coordonnées du point suivant en confondant la trajectoire avec le cercle de courbure.

Le calcul se poursuit jusqu'à ce que la trajectoire touche la surface de la pointe. Le point d'impact, repéré par son angle polaire $\varepsilon$ avec l'axe de la pointe, ainsi que l'angle d'incidence $\omega$ de la trajectoire sur la surface, sont alors déterminés.

3.3 DESCRIPTION DES ABAQUES. - La géométrie choisie (cf. Fig. 1) correspond à une situation expérimentale courante : la direction du faisceau incident de particules en l'absence de champ est perpendiculaire à l'axe de la pointe. Le facteur de forme est $f=0,15$. D'après la propriété fondamentale énoncée au paragraphe 1 , les angles $\omega$ et $\varepsilon$ ne dépendent que du point de départ et du paramètre $m v_{0}^{2} / \alpha E_{0}^{2}$ pour une géométrie donnée. Nous pouvons donc tracer les courbes donnant $\omega$ en fonction de $\varepsilon$ (Fig. 2), ainsi qu'un abaque 


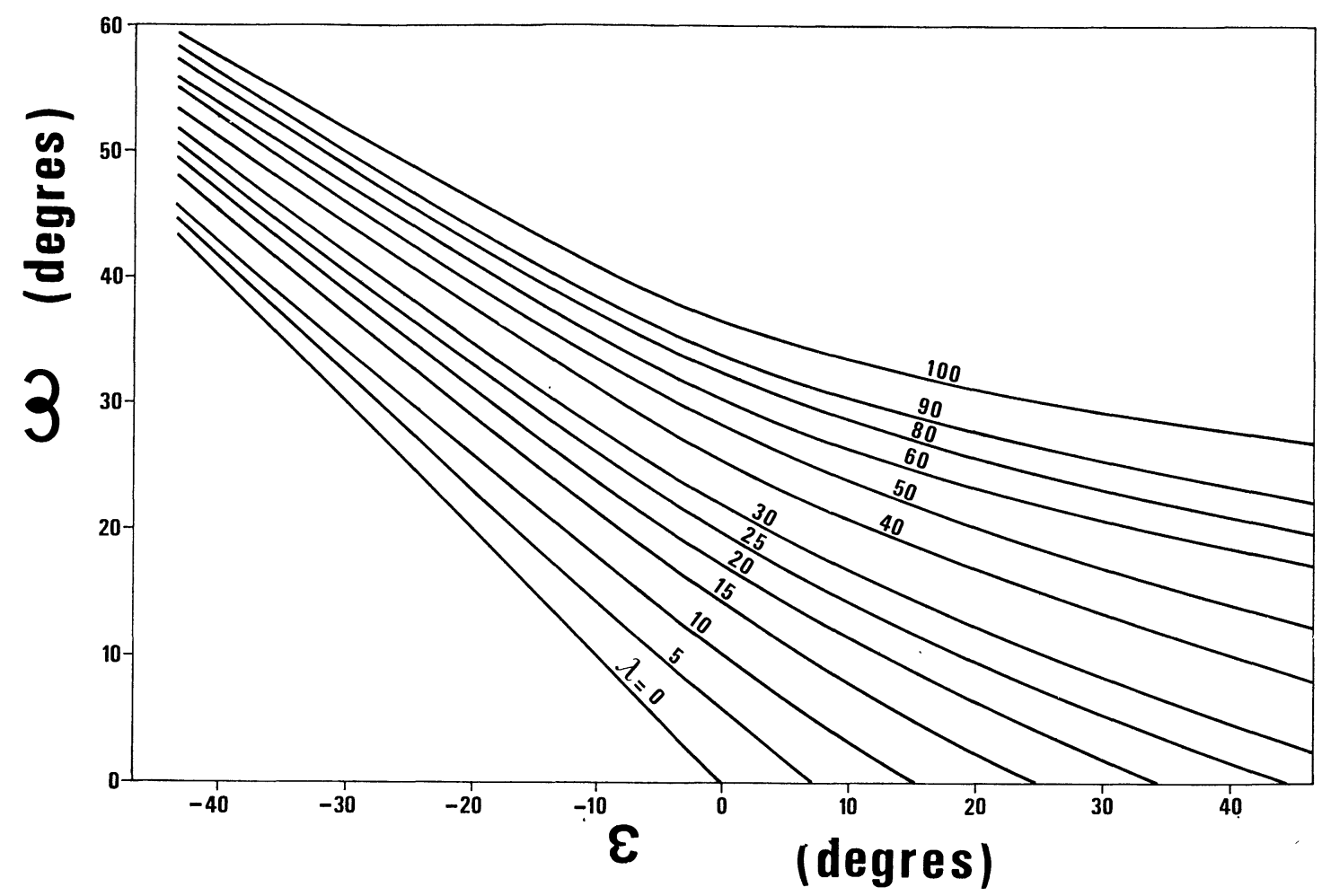

Fig. 2. - Angle $\omega$ en fonction de l'angle $\varepsilon$ pour différentes valeurs du paramètre $\lambda$.

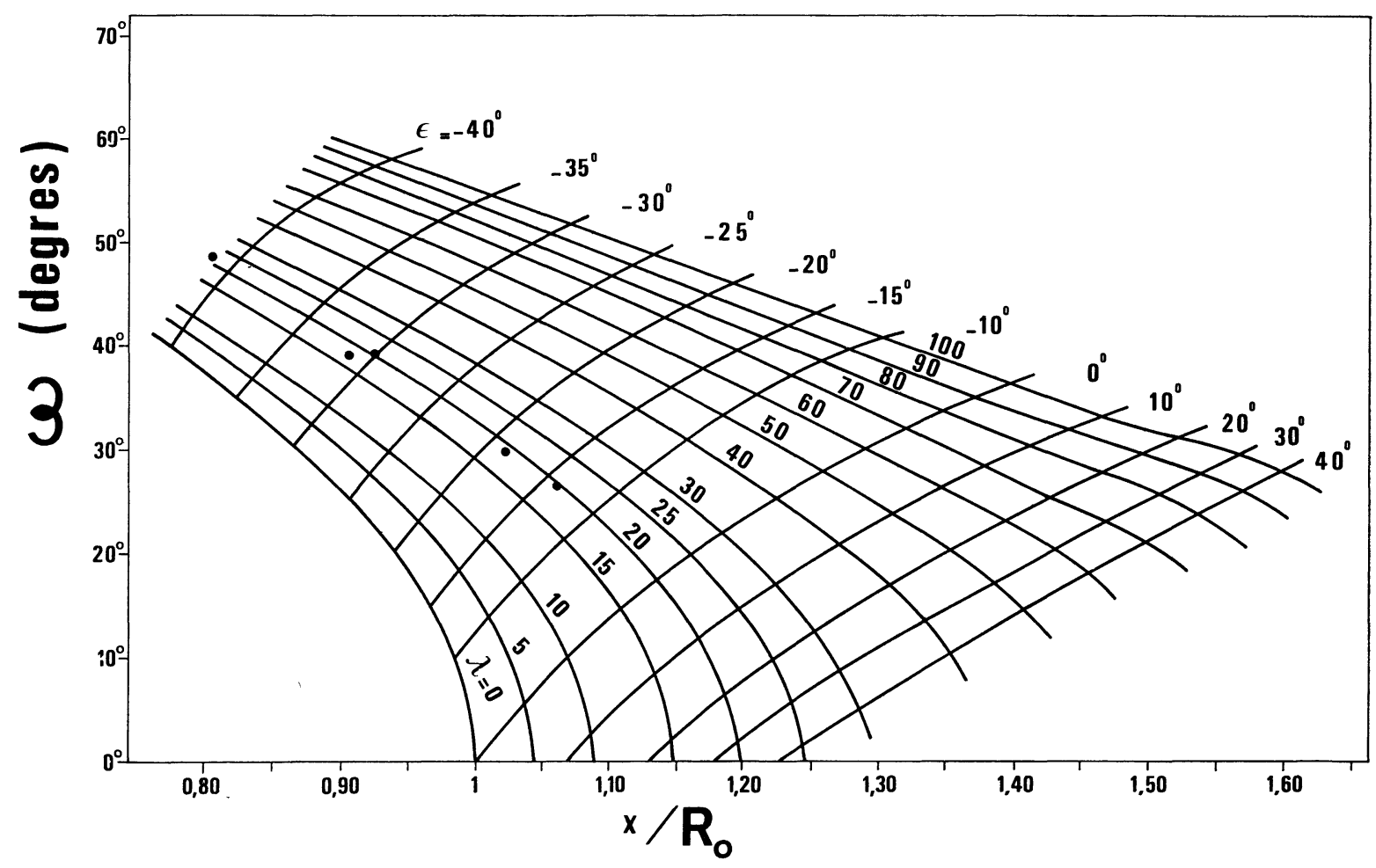

FIG. 3. - Angles $\omega$ et $\varepsilon$ en fonction du point de départ des trajectoires, pour différentes valeurs de $\lambda$. Les points sont les valeurs expérimentales correspondant au tableau donné dans le texte. 
donnant les valeurs de $\omega$ et $\varepsilon$ en fonction du point de départ, pour diverses valeurs de ce paramètre (Fig. 3). Pour des raisons d'utilisation pratique, les courbes sont tracées pour différentes valeurs de :

$$
\lambda=207 \frac{\alpha E_{0}^{2}}{m v_{0}^{2}}
$$

avec $E_{0}$ en $V / \AA$, $v_{0}$ en $\mathrm{km} / \mathrm{s}, \alpha$ en $\AA^{3}$ et $m$ en unités de masse atomique. L'angle $\varepsilon$ est négatif si le point d'impact de la particule sur la surface est du côté du faisceau incident, positif s'il est dans la zone d'ombre. Pour la détermination de $\omega$, nous avons confondu la surface de la pointe avec une sphère de centre $C$ (cf. Fig. 1) et de rayon $R_{0}$. L'erreur est inférieure à $2^{\circ}$ pour $\varepsilon<40^{\circ}$ et $f>0,1$, ce qui justifie l'approximation, compte tenu des erreurs de mesure (cf. $\S 4$ ).

3.4 GÉNÉRALITÉ DES ABAQUES. - Les abaques des figures 2 et 3 sont tracés pour des valeurs particulières du facteur de forme et du rayon de courbure au sommet de la pointe. En outre, on s'est limité aux trajectoires situées dans le plan de symétrie défini par l'axe de la pointe et la direction des trajectoires. Pour une utilisation générale, l'influence de ces divers facteurs doit être discutée.

Les facteurs de forme des pointes habituellement fabriquées varient de 0,1 à 0,2 . Les calculs effectués pour ces deux valeurs de $f$, avec le mêmo champ au sommet de la pointe, donnent des résultats à peu près identiques aux précédents (à environ $2^{\circ}$ près). Compte tenu des erreurs dans les mesures, discutées au paragraphe 4.6, les figures 2 et 3 doivent donc être valables pour toutes les pointes ayant un facteur de forme compris entre 0,1 et 0,2 . Le rayon $R_{0}$ de la pointe peut varier en pratique, de $1000 \AA$ à $10000 \AA$. Pour ces deux valeurs extrêmes, les points de départ des trajectoires se trouvent dans une région où elles sont pratiquement rectilignes. Pour la même valeur du champ au sommet, on retrouve alors des trajectoires géométriquement semblables.

Le problème réel est un problème à trois dimensions : des trajectoires situées en dehors du plan de symétrie pour lequel ont été faits les calculs, présentent une torsion et tendent à se rapprocher de ce plan (phénomène de convergence). Le flux incident sur une face cristalline située dans le plan de symétrie va donc être augmenté. Pour évaluer l'importance de ce phénomène, nous avons calculé la composante de la force normale au plan de symétrie en différents points de l'espace situés hors de ce plan. Ce calcul montre que la variation de flux due à ce phénomène doit être négligeable pour des points d'impact situés à moins de $10^{\circ}$ de la trace du plan de symétrie sur la pointe.

En conclusion, les abaques présentés doivent être utilisables pour des faces cristallines situées dans une région d'environ $20^{\circ}$ de largeur totale, s'étendant de part et d'autre du plan défini par l'axe de la pointe et la direction du jet, et cela pour la majorité des formes de pointes rencontrées en pratique.
4. Application à la mesure de la polarisabilité. 4.1 DEGRÉ DE RECOUVREMENT ET TEMPS DE DÉPOT. En l'absence de champ électrique, les particules d'un jet monocinétique de vitesse $\mathbf{v}_{0}$ et de densité $j_{0}$, arrivent sur la pointe du microscope à émission de champ en un faisceau pratiquement parallèle. Sur une face individuelle, repérée par l'angle $\varepsilon$ de sa normale avec l'axe de la pointe (Fig. 1) l'angle d'incidence des particules est $\omega_{0}$; le temps $\tau_{0}$ nécessaire pour atteindre un degré de recouvrement donné est inversement proportionnel au flux incident (si ce flux est constant) :

$$
\frac{1}{\tau_{0}} \sim j_{0} \sin \omega_{0} .
$$

En présence de champ électrique, les trajectoires sont courbées, la densité des particules dans le faisceau est $j$ et leur angle d'incidence sur la même face est $\omega$; il faut maintènant le temps $\tau$ pour atteindre le même degré de recouvrement :

$$
\frac{1}{\tau} \sim j \sin \omega
$$

Ceci suppose que le coefficient de collage est le même avec et sans champ. Cette hypothèse semble justifiée, car on opère toujours dans des conditions où le coefficient de collage des particules sur la surface est égal à 1 en l'absence de champ [7].

On a vu que l'angle d'incidence $\omega$ sur une face donnée ne dépend que du paramètre $\lambda$; par conséquent on a :

$$
\frac{\tau_{0}}{\tau}=\frac{j}{j_{0}} \frac{\sin \omega(\lambda)}{\sin \omega_{0}} .
$$

4.2 Divergence Des TRAJECTOIRES. - Deux trajectoires initialement parallèles et distantes au départ de $\Delta x$, touchent la surface de la pointe en deux sites définis respectivement par $\varepsilon$ et $\varepsilon+\Delta \varepsilon$. Il en résulte que la densité $j(\varepsilon)$ des particules sur la surface en présence de champ est :

$$
j(\varepsilon)=D(\varepsilon) \cdot j_{0}(\varepsilon) .
$$

Le facteur $D$ est un terme correctif qui vaut :

$$
D(\varepsilon)=\frac{\Delta x}{2 \sin \frac{\Delta \varepsilon}{2} \cdot \sin \omega} .
$$

Avec $\Delta x=50 \AA$, nous avons déterminé numériquement $\Delta \varepsilon$ pour différentes trajectoires, et calculé les valeurs correspondantes de $D(\varepsilon)$. Le calcul montre que $D$ est pratiquement indépendant de $\lambda$. La figure 4 donne la quantité $1 / D(\varepsilon)$ en fonction de $\varepsilon$.

4.3 ProcédÉ DE MESURE DE LA POLARISABILITÉ. Le facteur de forme $f$ et le rayon de courbure au sommet do la pointe $R_{0}$ sont d'abord déterminés expérimentalement [6] ; on en déduit la valeur du champ au sommet $E_{0}$, en mesurant le voltage nécessaire pour obtenir un courant d'émission de champ donné. On 


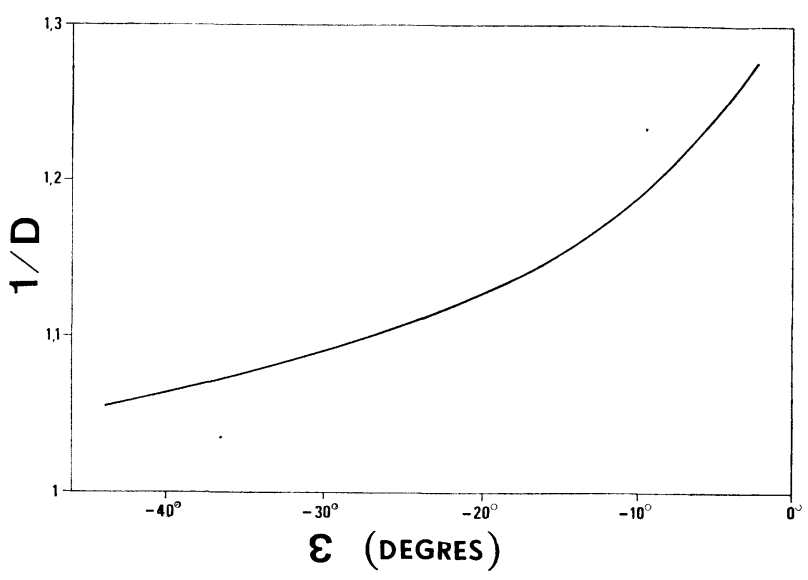

FIG. 4. - Inverse du facteur correctif $D$ en fonction de l'angle $\varepsilon$.

choisit ensuite sur l'image donnée par la pointe une face monocristalline située dans la zone qui s'étend sur une largeur de $\pm 10^{\circ}$ de part et d'autre du plan défini par l'axe de la pointe et la direction du jet atomique. Le choix de la face fixe l'angle $\varepsilon$. On détermine les temps de dépôt $\tau$ et $\tau_{0}$ nécessaires pour obtenir un même degré de recouvrement sur cette face, avec et sans champ électrique, en mesurant le courant d'émission local. Cette détermination se fait de préférence, dans un domaine où la variation du travail de sortie de la face en fonction du degré de recouvrement est relativement importante.

Les équations (6) et (7) permettent de calculer la valeur de l'angle $\omega$,

$$
\sin \omega(\lambda)=\frac{\tau_{0}}{\tau} \cdot \frac{1}{D(\varepsilon)} \cdot \sin \omega_{0} .
$$

Le paramètre $\lambda$ est alors déterminé à l'aide de l'abaque présenté à la figure 3 . La mesure peut être faite sur plusieurs faces individuelles, c'est-à-dire pour plusieurs valeurs de l'angle $\varepsilon$; naturellement, on doit retrouver toujours la même valeur du paramètre $\lambda$, ce qui permet de contrôler la mesure. Connaissant $E_{0}, v_{0}$ et $m$, la polarisabilité $\alpha$ est obtenue à partir de la formule (5) :

$$
\alpha=\frac{\lambda m v_{0}^{2}}{207 E_{0}^{2}} .
$$

4.4 INFLUENCE DE LA DISTRIBUTION DES VITESSES. Les jets thermiques de particules ne sont que très approximativement monocinétiques. Pour des valeurs données de $\alpha, E_{0}$ et $m$, nous avons calculé numériquement les valeurs du rapport $\tau / \tau_{0}$, d'une part, en admettant que toutes les particules ont la même vitesse égale à la vitesse la plus probable dans le jet, d'autre part, en tenant compte de la répartition des vitesses. Les résultats diffèrent de moins de $5 \%$. On peut donc se contenter de l'approximation de la vitesse la plus probable pour déterminer la polarisabilité, approximation déjà faite dans les méthodes précédentes [3].

4.5 EXEMPLE DE MESURE : POLARISABILITÉ DE L'ATOME DE PLOMB. - A titre d'exemple, nous avons appliqué cette méthode à la mesure de la polarisabilité du plomb. Le microscope électronique de champ est équipé d'un cylindre de Faraday pour mesurer le courant émis par des faces individuelles [7] et d'une source de plomb qui donne un jet thermique arrivant sur la pointe perpendiculairement à son axe. On mesure avec et sans champ électrique, le temps nécessaire pour atteindre, par exemple, le minimum du courant d'émission de champ (c'est-à-dire pour déposer un degré de recouvrement bien défini) sur diverses faces monocristallines. Le tableau ci-dessous résume les résultats expérimentaux :

$$
m=207 ; \quad v_{0}=0,317 \mathrm{~km} / \mathrm{s} ; \quad E_{0}=0,32 v / \AA \text {. }
$$

$\begin{array}{ccccccc}\text { Faces } & \varepsilon & \tau_{0} / \tau & \begin{array}{c}\text { Angle de la } \\ \text { avec le plan de } \\ \text { symétrie }\end{array} \\ \overline{(13 \overline{2})} & -4^{\circ} 54^{\prime} & 1,08 & - & \omega & \lambda \\ (13 \overline{1}) & -31^{\circ} 29^{\prime} & 1,11 & 13^{\circ} & 48^{\circ} 40^{\prime} & (22) \\ (12 \overline{1}) & -30^{\circ} & 1,16 & 4^{\circ} & 39^{\circ} & (17,5) \\ (23 \overline{1}) & -19^{\circ} 6^{\prime} & 1,35 & 9^{\circ} & 39^{\circ} 10^{\prime} & 20 \\ (34 \overline{1}) & -13^{\circ} 54^{\prime} & 1,60 & 5^{\circ} & 29^{\circ} 50^{\prime} & 19 \\ & & & 4^{\circ} & 26^{\circ} 40^{\prime} & 20\end{array}$

Les points correspondants à ce tableau sont représentés sur la figure 3.

4.6 Discussion. - Les valeurs de $\lambda$ trouvées sont voisines de la valeur moyenne de 19,7 , ce qui montre que notre mesure est correcte. Deux causes d'erreur peuvent affecter la détermination de $\lambda$. D'une part une mesure sur une face trop éloignée du plan de symétrie, comme ici la face (132), augmente la valeur de $\tau_{0} / \tau$ (convergence des trajectoires dans l'espace, cf. $\S 3.5$ ) et par suite la valeur de $\lambda$. D'autre part, $\tau_{0} / \tau$ est mesuré à 3 ou $4 \%$ près dans nos expériences. Une erreur de $4 \%$ sur ce rapport entraîne une erreur sur $\lambda$ supérieure à $15 \%$ pour $\varepsilon>30^{\circ}$ et de $10 \%$ pour $\varepsilon \simeq 15^{\circ}$. Il faut donc utiliser les mesures faites sur les faces les plus proches de l'apex. En outre, pour ces faces $\tau_{0} / \tau$ est mesuré avec la meilleure précision ( $3 \%$ ici). Si on fait

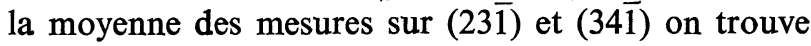
$\lambda=19,5$ avec une erreur relative inférieure ou égale à $10 \%$.

L'incertitude sur $\alpha$ provient des erreurs sur $\lambda, v_{0}^{2}$ et $E_{0}^{2}$. Le jet de plomb provient d'une source équivalente à une cellule de Knudsen à la température $T$. La vitesse la plus probable dans le jet est donc telle que $m v_{0}^{2}=3 k T$. L'erreur relative sur $v_{0}^{2}$ est donc égale à l'erreur relative sur $T$, soit moins de $2 \%$. Le champ au sommet $E_{0}$ n'est pas mesuré à moins de $10 \%$ près [6]. Compte tenu de l'erreur sur $\lambda$, déjà discutée, la valeur de $\alpha$ est donc connue à $30 \%$ près. Au total, pour le plomb,

$$
\alpha=19,5 \pm 6 \AA^{3} \text {. }
$$

La précision, bien que faible, est quand même supérieure à celle obtenue par les anciennes méthodes $[3,4]$, et surtout évaluée avec plus de certitude. En effet, la forme de la pointe est prise en compte dans nos 
abaques et un degré de recouvrement bien déterminé est repéré avec précision dans la mesure de $\lambda$. L'erreur principale sur $\alpha$ provient de l'incertitude dans la détermination de $E_{0}$, incertitude qui existait déjà dans les autres méthodes et qui était probablement très supérieure à $10 \%$. Il est en principe possible d'améliorer la mesure de $E_{0}$, par exemple en déterminant la forme de la pointe dans un microscope électronique à balayage. De même on peut diminuer l'erreur sur $\lambda$ en augmentant la précision sur $\tau$ et $\tau_{0}$. Nous pensons qu'on peut ainsi réduire l'erreur relative sur $\alpha$ à moins de $20 \%$.

Des valeurs relatives de la polarisabilité peuvent être déterminées avec une plus grande précision. Pour cela, il faut placer deux sources de jet atomique différentes près d'une pointe d'émission de champ, et effectuer la mesure en utilisant chaque jet individuellement. Avec le même champ $E_{0}$ dans les deux cas, on obtient :

$$
\frac{\alpha_{1}}{\alpha_{2}}=\frac{\lambda_{1} m_{1} v_{01}^{2}}{\lambda_{2} m_{2} v_{02}^{2}} .
$$

L'erreur doit être alors inférieure à $20 \%$.

A notre connaissance, c'est la première mesure de la polarisabilité de l'atome de plomb libre. On sait que l'ordre de grandeur de $\alpha$ peut être estimé à partir du volume atomique [8]. Pour le cas du plomb, on obtient $22 \AA^{3}$ pour le volume, ce qui est en bon accord avec notre mesure. Il existe des évaluations théoriques de $\alpha$, soit à partir des fonctions d'ondes atomiques de Hartree-Fock [9], soit à partir de fonctions d'ondes plus simples [10]. Pour le plomb on calcule ainsi $\alpha=11,7 \AA^{3}$ dans le premier cas, et $\alpha=6,28 \AA^{3}$ dans le second. Le choix des fonctions utilisées par le calcul est donc assez critique, au moins pour l'atome de plomb. Il semble préférable d'utiliser les valeurs expérimentales de $\alpha$ pour tester les approximations théoriques [10].

5. Conclusion. - La méthode de Drechsler et Müller pour la mesure de la polarisabilité $\alpha$ d'atomes libres a été reprise et améliorée. D'une part une propriété générale des trajectoires a été établie et utilisée pour la construction d'abaques qui donnent les propriétés des trajectoires dans le champ électrique au voisinage de la pointe d'un microscope électronique de champ. Les formes de pointe les plus couramment utilisées en pratique sont considérées dans ces abaques. Ainsi, on évite la construction point par point des trajectoires dans chaque cas particulier. D'autre part, une méthode simple est donnée pour une détermination expérimentale plus précise de $\alpha$.

En principe, la méthode s'applique à toutes les particules pouvant être produites en jets atomiques et dont l'adsorption sur la pointe provoque une modification du travail de sortie de la surface. Dans un premier essai d'application, on a trouvé pour l'atome de plomb $\alpha=19,5 \pm 6 \AA^{3}$, valeur qui semble raisonnable. Il est possible de diminuer l'erreur encore importante en améliorant la mesure du champ électrique à l'extrémité de la pointe. Telle qu'elle est la méthode doit permettre d'obtenir rapidement les polarisabilités des métaux de transition, qu'il est très utile de connaître, en particulier pour l'explication des phénomènes de désorption par effet de champ.

\section{Bibliographie}

[1] Scheffers, H. et STark, J., Phys. Z. 35 (1934) 625.

[2] Scheffers, H., Phys. Z. 41 (1940) 399.

[3] Drechiler, M. et Muller, E. W., Z. Phys. 132 (1952) 195.

[4] LIEPACK, H. et DrechSLER, M., Naturwissenschaften 3 (1956) 52.

[5] Muller, E. W. et Tsong, T. T., Field Ion Microscopy. Principles and Applications. (American Elsevier N. Y.) 1969 p. 25.
[6] Drechsler, M. et Henkel, E., Z. Angew. Phys. 6 (1954) 341. [7] Bermond, J. M. et Bel, F., Revue Phys. Appl. 5 (1970) 649.

[8] Gyftopoulos, E. P. et Levine, J. D., J. Appl. Phys. 33 (1962) 67.

[9] Fraga, S., Karwowski, J. et Saxena, K. M. S., Atmoic Data and Nuclear Data Tables 12 (1973) 467.

[10] Thorhallson, J., Fisk, C. et Fraga, S., J. Chem. Phys. 49 (1968) 1987. 